\title{
The Clinical Significance and Diagnostic Value of Echogenic Intracavitary Foci in Fetal Heart
}

\author{
Li Kaiwen \\ Department of Ultrasound, Jingzhou Central Hospital, The Second Clinical Medical College, Yangtze University, Jingzhou, China
}

Email address:

likaiwen1234@163.com

To cite this article:

Li Kaiwen. The Clinical Significance and Diagnostic Value of Echogenic Intracavitary Foci in Fetal Heart. Clinical Medicine Research. Vol. 7, No. 2, 2018, pp. 40-42. doi: 10.11648/j.cmr.20180702.11

Received: March 27, 2018; Accepted: April 11, 2018; Published: May 11, 2018

\begin{abstract}
This paper aims at investigating the clinical significance and diagnostic value of fetal echocardiography in the detection of echogenic intracavitary foci (EIF). Then, it analyzed the ultrasonic features of the intensive echocardiography from June 2016 to June 2017, which were found in 535 mid-term (20-26 weeks) fetuses, and performed the echocardiographic follow-up during the third trimester and after birth. As a result, in 535 fetuses, 502 cases of late pregnancy (35 40 weeks) showed that the echo in the intracardiac hyperechogenic focus declined or disappeared. The intracardiac echo foci still existed in 33 cases after birth, which disappeared in 3 6 months. Finally, it is concluded that fetal intracerebral echogenic lesions are mostly transient ultrasonography with favorable prognosis.
\end{abstract}

Keywords: Clinical Significance, Diagnostic Value, Echogenic Intracavitary Foci (EFI), Fetal Heart

\section{Introduction}

Prenatal ultrasound examination is one of the essential auxiliary examinations for detecting fetal abnormalities. With the continuous improvement of ultrasound instruments and the development of examination techniques, the detection rate of fetal echogenic intracavitary foci (EFI) is increasing [1-2]. The significance of its existence still nags clinicians and it also brings many mental burdens to pregnant women and their families. In this study, echocardiography was used to detect and assess the risk of fetal ElF, and its significance in clinical detection of fetal developmental abnormalities was discussed.

\section{Materials and Methods}

\subsection{Research Object}

Selected from those who performed fetal echocardiography in our hospital from June 2016 to June 2017, 535 pregnant women were identified with strong echo foci in their ventricular cavities. The gestational age was from 20 to 26 weeks, with an average of 23.5 weeks. The age of pregnant women was from 19 to 41 years old, with an average age of 28.7 years.

\subsection{Instruments and Methods}

The ultrasound instrument used was a GE Voluson E8 with a probe frequency of 2.0-5.0 $\mathrm{MHz}$. The two-dimensional and flow Doppler examination of the fetal heart section was mainly based on the clinical guidelines issued by the American Institute of Ultrasound in Medicine (AIUM). [3]. The conventional section of the fetal heart examination was used in fetal heart examination. Scanning the fetal head and spine to determine the position; The cross-section of abdomen's stomach bubble was used to determine the positional relationship between the heart and the viscera and the apical direction. In the fetal chest section, the apical four-chamber view, left ventricular outflow tract, right ventricular outflow tract, three-vessel section and three-vessel tracheal section were shown. The probe is parallel to the long axis of the fetus to display the aortic arch section and the arterial arch section. Observe atrioventricular cavity ratio, foramen ovale, oval flap, flap artery and atrioventricular valve and so on in the above sections. Whether there is a defect in the atria and ventricle should be studied. The connection between the vein and the 
atrium and the connection between the atria and the ventricle should be observed and the abnormality of the connection between the ventricle and the aorta. Observing whether there are abnormal echoes in each heart chamber and determine if there is a heart malformation. The hemodynamic changes of the fetal heart were observed by superimposing color blood flow on the basis of a clear image. If necessary, local magnification and film replay can be used to make the fetal heart anatomical structure more clear. The routine echocardiographic examination of the heart was used to examine the cardiac follow-up of newborns.

\section{Results}

\subsection{ElF Distribution and Echocardiographic Performance}

It is investigated that the fetus with EIF accounts for approximately $8.1 \%$ of the fetus in the same period. Among the 535 fetuses, $489(91.4 \%)$ were located in the left ventricle, and most of the EIF were in the papillary muscles and chordate; 20 cases $(3.7 \%)$ were located in the right ventricle (17 of them were in the right ventricular bundle and 3 in the right ventricular apex); 26 cases $(4.9 \%)$ were located in bilateral ventricles. See Table 1.

Table 1. Location of EIF distribution in the fetus.

\begin{tabular}{llllll}
\hline \multirow{2}{*}{ Number of cases scope $(\mathbf{m m})$} & \multicolumn{2}{l}{ Left ventricle } & \multicolumn{3}{l}{ Right ventricle } \\
\cline { 2 - 5 } & Solitary double & Solitary double & \\
\hline 535 & $2.7 \pm 1.2$ & 423 & 66 & 18 & 2 \\
\hline
\end{tabular}

The Fetal EIF echocardiography showed a patchy, foci-like or cord-like strong echo in the ventricular cavity of the fetus, with a diameter of 1.8 to $5.1 \mathrm{~mm}$. The echo intensity was comparable to that of the bone, but there was no obvious acoustic shadow. See Figure 1 and 2.

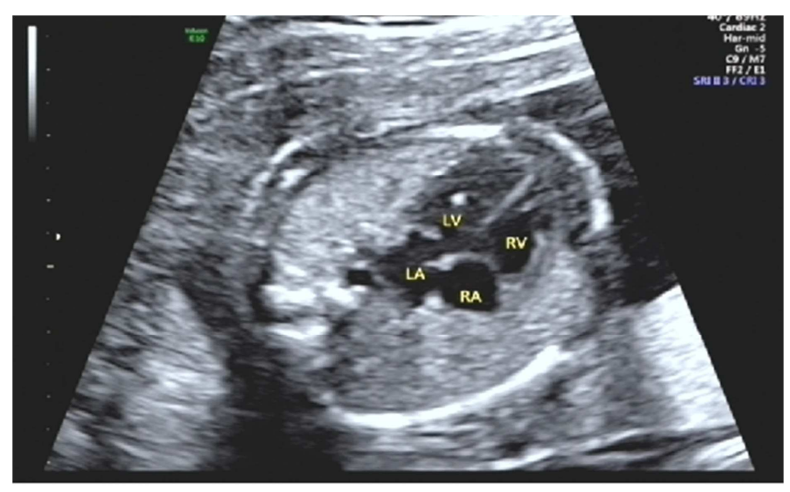

Figure 1. Left ventricular hyperechoicity.

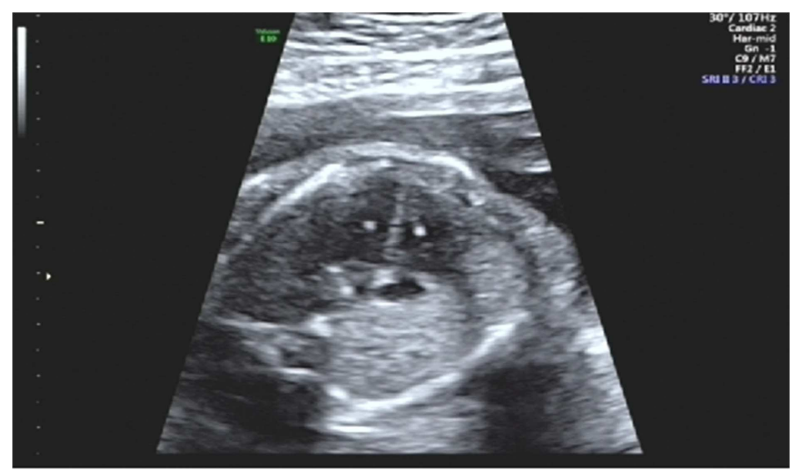

Figure 2. Biventricular hyperechoicity.

\subsection{Follow-up Survey}

Fetal EIF was detected and echocardiograms were performed before and after children's birth. In late pregnancy (35 to 40 weeks), 502 (93.8\%) of the 535 fetuses showed a decrease or disappearance of ElF echo intensity with increasing gestational age or prolonged time, and 33 cases
$(6.2 \%)$ of ElF could still be explored. Postnatal neonatal echocardiography showed that the heart, morphology, structure, and blood flow were normal, and EIF disappeared after 3 to 6 months of delivery.

\section{Discussion}

Under the impetus of the continuous development of medical technology, the resolution of color ultrasound has been rapidly improved, and it has gradually become one of the main means for clinicians to diagnose patients directly. Prenatal ultrasound has been widely used in clinical practice. At the same time, ultrasound technology has been significantly improved, and the detection rate of fetal ventricular strong echogenic lesions has been continuously rising. Many research scholars at home and abroad have conducted a comprehensive observation of the causes, but the reason for the occurrence of ventricular echogenic lesions is still not clear [4]. It has been declared in the literature that the detection rate of ElF in normal pregnant women is $2 \%$ to $5 \%$ [5]. In this paper, the detection rate of fetal EIF was $8.1 \%$, which was slightly different from the literature reports. There are some differences in the detection rate of fetal EIF in different literatures. The following factors are considered: the composition of the research object, the quality and resolution of the instrument, the technology and experience of the operative doctor, the position of the fetus, the condition of the pregnant woman itself, and the gestational age. The effects of fetal ElF on the fetal heart and the understanding of the EIF by clinicians are directly related to the treatment of pregnant women. Therefore, to clarify the clinical pathological value and prognosis of fetus ElF is of great significance for clinical decision-making.

The fetal ElF was first described by Allan in 1986 [6]. In recent years, domestic and foreign scholars have also made a lot of research reports, and found that its mechanism may be related to the following aspects: 1. strong echogenic reflections formed by limited thickening of the ventricle, such as chordae tendinae, papillary muscles, and displaced muscular bundles; 2 . mineral deposits and calcifications in the 
central part of the nipple; 3. abnormal development of microvascular may lead to early ischemic changes in the distal branches of the papillary muscles, resulting in micro calcification of the papillary muscles; 4 . it may be an incomplete perforation of the teat tendons, which can be a variation in normal atrial and ventriculum development.

In recent years, some scholars believe that fetal cardiac hyperechoic foci are associated with fetal chromosomal abnormalities. On the basis of relevant papers, the analysis conducted by Smith-Bindman et al. shows that fetal EIF is associated with trisomy 21 . The sensitivity of using fetal intracardiac echogenic lesions as markers for fetal chromosome screening is $11 \%$ [8]. It is also reported that Fetal EIF appears to increase the risk of fetal chromosomal abnormalities. [9].

At present, with the development of research and clinical practice, it is generally believed that the presence of EIF alone is not significant in the clinic. If other soft indexes are combined, such as fetal lateral ventricle widening, choroidal cysts, the posterior fossa effusion, strong intestinal echo, short femur or tibia, renal pelvis separation, or structural abnormalities, the risk of heart malformations and chromosomal abnormalities would increase. The presence of fetal EIF in low-risk populations has no clinical significance, but there is greater significance for warnings in high-risk populations [10]. Due to the limited conditions, no chromosome screening was performed in this study, and no obvious cardiac malformations and other structural abnormalities were found in 33 cases of follow-up fetuses.

As the gestational weeks progressed, most of the EIF echoes gradually weakened or disappeared. In this group, $93.8 \%$ of the fetus disappeared in the late pregnancy and $6.2 \%$ of the fetus disappeared at 3 6 months after birth. All the EIF fetuses in this study were not accompanied by congenital cardiovascular malformations, arrhythmia or other abnormalities. Therefore, the presence of EIF did not increase the risk of congenital heart defects.

It is necessary to identify multiple ventricular rhabdomyoma of the fetus when multiple EIF clusters are aggregated into a flaky strong echo or coexist in the left and right ventricles. In general, EIF is mostly single, which is connected with chordae tendineae and papillary muscles. The size is usually $<6 \mathrm{~mm}$, and it gradually weakens or disappears with time prolonging. The tumor is mostly multiple, and the echo or strong echo mass of the ventricular cavity, connected to the ventricular septum or ventricular wall, gradually increased [5].

When fetal EIF is found, different methods should be used to deal with different situations: As for the single and low risk population, ultrasound should be described, and no pathological diagnosis is recommended, and dynamic observation is suggested. As for the high-risk groups, multiple data should be combined, and comprehensive assessment of risk of cardiac malformation and chromosomal abnormalities should be made seriously. If combined with other soft indicators or structures are abnormal, careful fetal echocardiography should be performed to further exclude fetal cardiac malformations, and prenatal chromosome karyotype analysis is recommended.

\section{Conclusion}

This study is a small sample study. Although fetal echocardiography has been carried out in the second trimester of pregnancy (20 26 weeks gestation), late pregnancy (35 40 weeks gestation) and postnatal newborns, it is still necessary to further accumulate more cases to demonstrate. To sum up, prenatal echocardiography for detecting fetal EIF is usually temporary, with good prognosis and no obvious cardiovascular malformations or other fetal malformations. Fetal EIF should not be used as an indication for termination of pregnancy. However, the possibility of fetal cardiac malformation and chromosomal abnormalities will be increased if it is high risk or combined with other soft indicators and structures are abnormal. Especially in high-risk groups, echocardiographic follow-up should be performed after the birth of the fetus to eliminate possible cardiovascular abnormalities that could not be detected by echocardiography during the fetal period.

\section{References}

[1] Liang Yiqi. Comparative study of echocardiographic anatomy of fetal heart with or without cardiac chamber [J]. Imaging technology, 2016 (1):22-23.

[2] Xing Min, Yang self, Wan Mei. Echocardiographic detection of fetal ventricular echogenic plaques and postnatal tracking analysis [J]. Defense Medical Journal of South China, 2016, 30 (4): $250-252$.

[3] American Institute of Ultrasound in Medicine. AIUM practice guide-line for the performance of fetal echocardiography [J]. Ultrasound Med, 2013, 32 (6):1067-1082.

[4] Zhang Jing, Zhou Qichang, Zhang Ming et al. Study on the hemodynamic changes of fetal ventricular dysplasia by Doppler ultrasonography [J]. Chinese Journal of ultrasound imaging, 2012, 21 (4):296-299.

[5] He Yihua. Fetal echocardiography [M]. Beijing: People's Health Press, 2013:325.

[6] Allan LD . Mannual of fetal echocardiography [M]. London:MTP Press, 1986:134.

[7] Pei Lei. Analysis of the clinical significance of intraventricular hyperechoic foci in fetal B-ultrasound [J]. Chinese Journal of eugenics and genetics, 2010, 18 (11):102-104.

[8] Smith-Bindman R, HosmerW, Feldstein VA, et a1. Second-trimester ultrasound to detect fetuseswith Down syndrome:ameta-analysis [J]. JAMA, 2001, 285:1044-1055.

[9] Lu Qing, flanking, Ren Fei. Analysis of fetal ventricular echogenic plaque in 367 cases pregnancy outcome [J]. Journal of Youjiang Medical College for Nationalities, 2017, 39 (2): 121-122.

[10] Li Xuemei, Feng Xinheng, Li Jingfu. Clinical significance of prenatal echocardiographic fetal ventricular echo location [J]. Chinese oligogenics magazine, 2016, 24 (12):841 842. 